\title{
Clean Construction Protocol for the National Ignition Facility Beampath and Utilities
}

S.C. Sommer, I.F. Stowers, D.E. Van Doren, R.A. Predmore, and S.A. Stephenson

This article was submitted to the 48th Institute of Environmental Sciences and Technology, Anaheim, CA April 28 - May 1, 2002

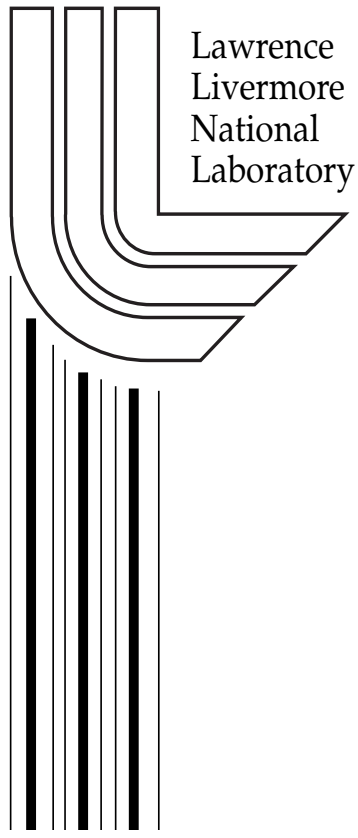

January 1, 2002 


\section{DISCLAIMER}

This document was prepared as an account of work sponsored by an agency of the United States Government. Neither the United States Government nor the University of California nor any of their employees, makes any warranty, express or implied, or assumes any legal liability or responsibility for the accuracy, completeness, or usefulness of any information, apparatus, product, or process disclosed, or represents that its use would not infringe privately owned rights. Reference herein to any specific commercial product, process, or service by trade name, trademark, manufacturer, or otherwise, does not necessarily constitute or imply its endorsement, recommendation, or favoring by the United States Government or the University of California. The views and opinions of authors expressed herein do not necessarily state or reflect those of the United States Government or the University of California, and shall not be used for advertising or product endorsement purposes.

This is a preprint of a paper intended for publication in a journal or proceedings. Since changes may be made before publication, this preprint is made available with the understanding that it will not be cited or reproduced without the permission of the author.

This report has been reproduced directly from the best available copy.

Available to DOE and DOE contractors from the Office of Scientific and Technical Information

P.O. Box 62, Oak Ridge, TN 37831

Prices available from (423) 576-8401

http://apollo.osti.gov/bridge/

Available to the public from the National Technical Information Service

U.S. Department of Commerce 5285 Port Royal Rd., Springfield, VA 22161

http://www.ntis.gov/

OR

Lawrence Livermore National Laboratory Technical Information Department's Digital Library http://www.llnl.gov/tid/Library.html 


\title{
CLEAN CONSTRUCTION PROTOCOL FOR THE NATIONAL IGNITION FACILITY BEAMPATH AND UTILITIES
}

\author{
Stanley C. Sommer, Lawrence Livermore National Laboratory \\ Irving F. Stowers, Lawrence Livermore National Laboratory \\ David E. Van Doren, Jacobs Facilities Incorporated \\ Robert A. Predmore, Lawrence Livermore National Laboratory \\ Sue A. Stephenson, Lawrence Livermore National Laboratory
}

\section{Biographies}

Stanley C. Sommer has been with the National Ignition Facility (NIF) Project since 1993 and currently is the Lawrence Livermore National Laboratory (LLNL) Clean Construction Protocol Manager and is responsible for developing and implementing the methodology for clean beampath installation in the Laser and Target Area Building (LTAB). A licensed Professional Engineer, with mechanical engineering degrees from Stanford University (MS, 1993) and University of California, Davis (BS, 1989), he is a member of the American Society of Mechanical Engineers and the Earthquake Engineering Research Institute.

Irving F. Stowers is a senior scientist in the NIF Program at LLNL where he has worked since 1975. His expertise includes laser and flashlamp damage to optics; design and construction of the Shiva, Nova, and NIF Laser Fusion Systems; fabrication of laser fusion targets; and the design and construction of ultra precision machine tools. He is the author of over 90 papers with more than 20 in the field of precision cleaning and contamination control. He received his BS in Mechanical Engineering from Northeastern University and his MS and ScD from Massachusetts Institute of Technology.

David E. Van Doren, Clean Construction Protocol Manager for Jacobs Facilities Incorporated (JFI), joined the NIF project in 1997. He is responsible for overseeing all clean construction protocol activities and implementing the specification requirements in the LTAB. He has 30 years experience in construction, 18 of those years solely dedicated to the construction of cleanrooms around the world. He has been the project manager for large semiconductor wafer fabrication plants and construction manager for pharmaceutical manufacturing facilities.

Robert A. Predmore, President of Predmore Project Management and consultant to LLNL, joined the NIF team in 1999 as the Beampath Infrastructure Systems Construction Manager. A licensed Professional Engineer, his BS is in Architectural Engineering from California State Polytechnic University (1971).

Sue A. Stephenson has ten years experience writing about laser technology at LLNL. She received her BA in Political Science from the University of Michigan and her MA in Communications from the University of California at Berkeley.

\begin{abstract}
When the stadium-size, National Ignition Facility (NIF) is fully operational at the Lawrence Livermore National Laboratory (LLNL), its 192 laser beams will deliver 1.8 megajoules (500 terawatts) of energy onto a target to create extremely high temperatures and pressures for inertial confinement fusion research. Due to the high-energy-physics requirements of the NIF optical components, the optics and their surrounding beampath as well as the supporting utility systems must be fabricated, cleaned, assembled, and commissioned for precision cleanliness. This paper will provide an overview of the NIF
\end{abstract}


cleanliness requirements, the clean construction protocol (CCP) specifications for the beampath and clean utilities, and techniques for verifying the CCP specifications.

The NIF cleanliness requirements define limits for molecular and particulate contamination; the goal of these limits is to prevent contamination of the optical components. To prevent laser-induced damage and poor laser quality in the optical components, requirements for cleaning, assembly, installation, and commissioning in terms of particle and nonvolatile residue (NVR) levels are defined. The requirements in the interior of the beampath are parts-per-billion airborne molecular contamination (AMC) and Class 1 particulate levels.

To achieve the cleanliness requirements for the beampath interior, a graded CCP approach is used as the NIF beampath and utilities are being constructed by a partnership between LLNL and the construction contractor, Jacobs Facilities, Inc. in a stadium-size Class 100,000 building. Installation of the beampath components utilizes localized mini-environments of Class 100 or better, with budgets of cleanliness exposure or "class-hours" for each clean connection. Garment, equipment, and operational considerations are evaluated with process verification.

Verification of the beampath and utility cleanliness is performed with cleanliness exposure monitoring, evaluating particulates with "swipes" and the LLNL-developed Precision Cleanliness Verification System (PCVS), and measuring NVRs and AMCs with analytical chemistry techniques. Preliminary cleanliness verification indicates that the CCP specifications are achieving the NIF cleanliness requirements for the beampath and clean utilities.

\section{Key Words}

Contamination control

Clean Construction Protocol (CCP)

Particulate swipe

\section{Introduction}

The largest U.S. science project currently under construction, NIF is being built at LLNL for the National Nuclear Security Administration within the U.S. Department of Energy. Construction of the Laser and Target Area Building (LTAB), shown in Figure 1, was completed in late 2001 and is comprised of two large laser bays, two tall optical switchyards, and a cylindrical target bay designed as Class 100,000 spaces. Figure 2 shows the major beampath components within the LTAB, such as bussize vacuum vessels, reinforced concrete support structures, relatively thin-walled interstage enclosures, and up to 25-meter-long beam tubes. These components enclose the NIF experiments and provide a controlled environment of clean dry air, argon gas, or vacuum, depending on the function of a particular portion of the beampath. While the spatial filters and target chamber are vacuum vessels, the remaining beampath enclosures contain clean dry air in the laser bays and argon gas in the switchyards and target bay.

NIF will be the largest optical instrument in the world containing 30,000 small optics and 7,360 large optics. While the small optics are typically circular and less than 15 centimeters in diameter, the large optics are nearly square and about 40 centimeters long on each side. The large optics consist of almost 3,000 pieces of laser glass, 1,800 vacuum windows and lenses, 1,600 mirrors and polarizers, 600 crystals, and 400 optical shields. NIF also contains more than 9,000 flashlamps to pump the solid state neodymium glass laser. 


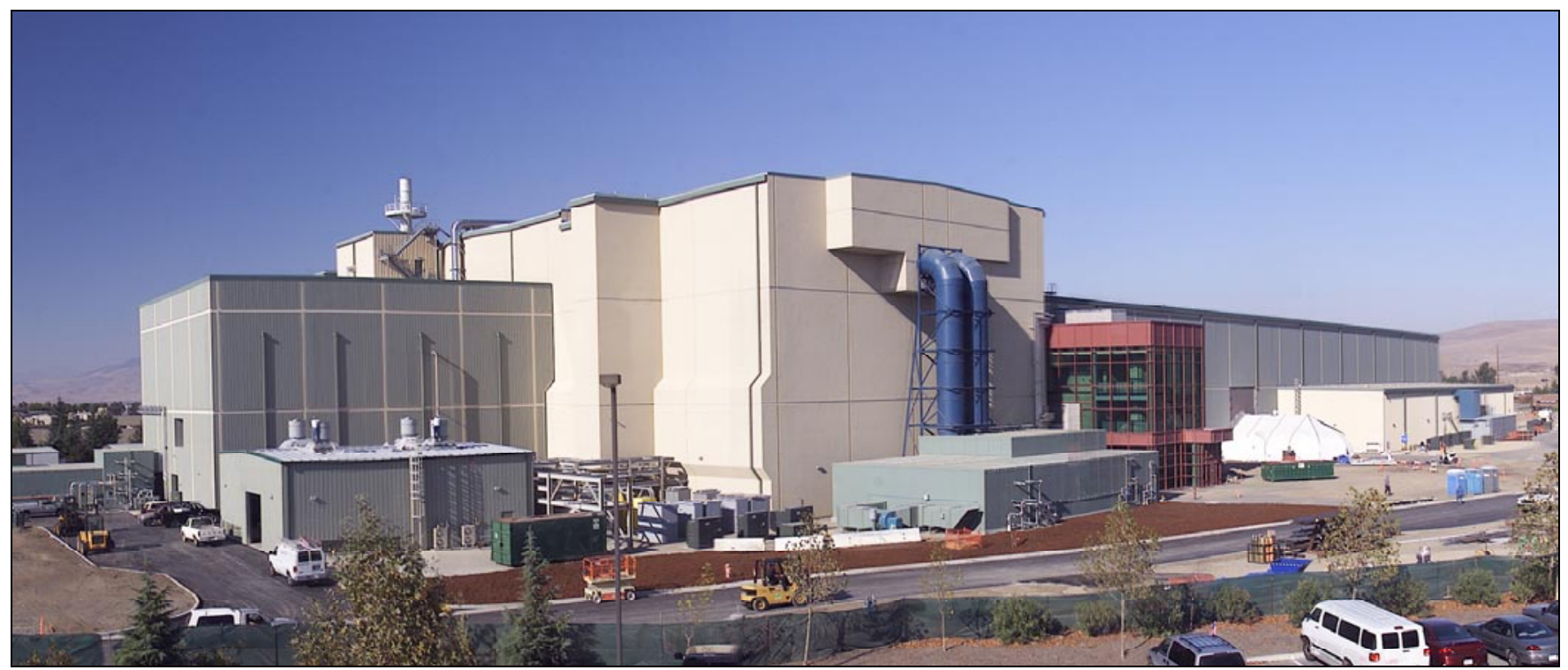

Figure 1. View of the National Ignition Facility (NIF) Laser and Target Area Building (LTAB).

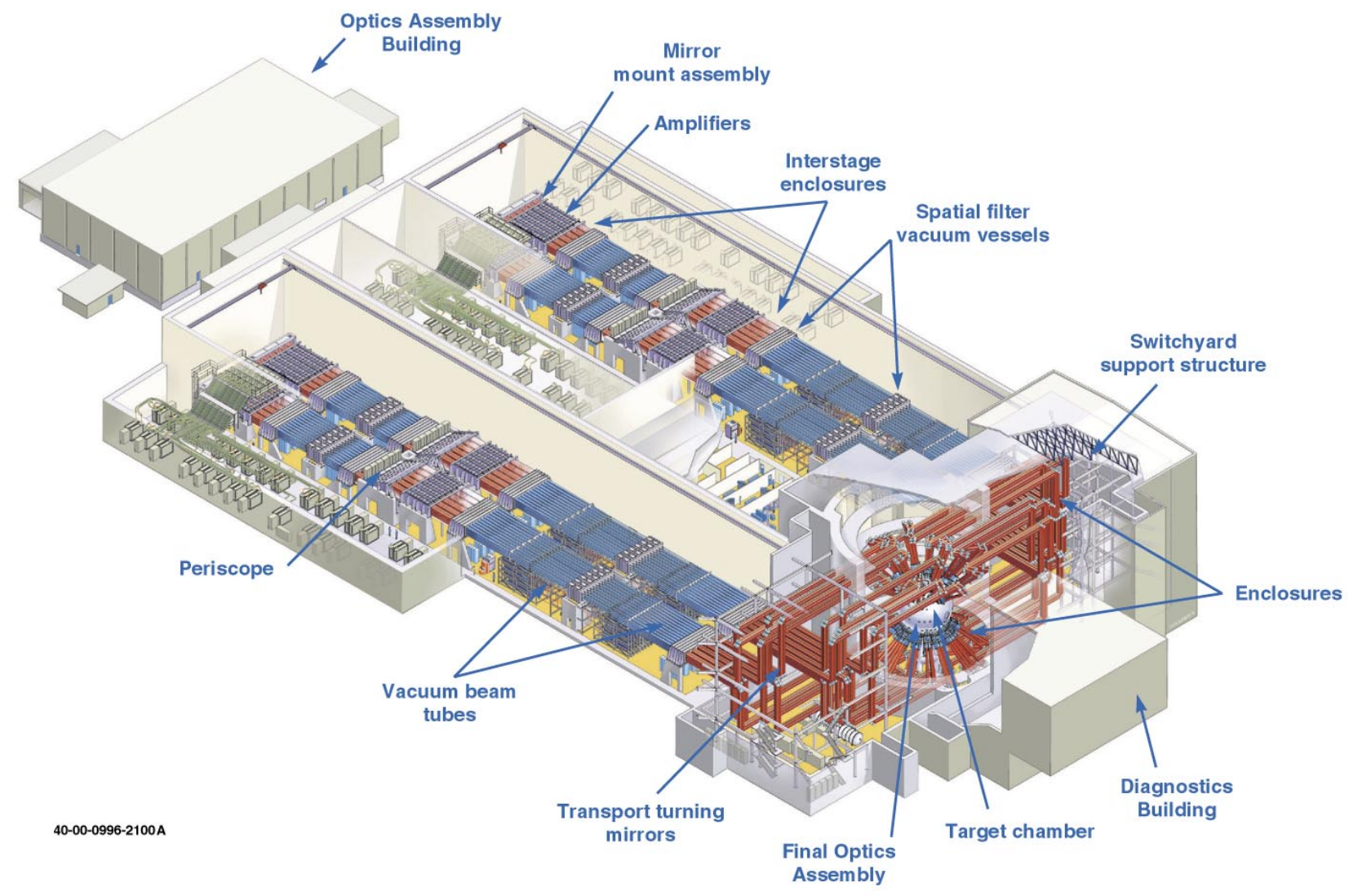

Figure 2. NIF beampath components.

To create the pressure and temperature conditions for inertial confinement fusion (ICF) research, NIF has up to 192 laser beams that can deliver 1.8 megajoules of energy into the ICF target. Each NIF laser beam is a short pulse of light about 400 millimeters (15 inches) square and from 100 to 6,000 millimeters (four inches to twenty feet) in length. A master oscillator generates the initial laser pulse 
using a neodymium-doped optical fiber ring that fits in the palm of a hand. The laser light is amplified and split until there are 192 beams at a few joules. Each laser beam is then injected into the large aperture beampath where it is multi-passed and amplified to more than 15 kilojoules. On the 1.5 microsecond journey along the approximately 180 meter (600 feet) of beampath, each laser pulse bounces off the equivalent of 54 mirrors and passes through approximately 2 meters ( 7 feet) of glass before reaching the target. At the outer edge of the evacuated target chamber, each laser pulse passes through potassium dihydrogen phosphate (KDP) crystals that convert the light from infrared (1.05 micrometers) to ultraviolet (0.35 micrometers) light. This light is then focused onto a millimeter-sized target where temperatures reach those found in stars and in nuclear weapons. The 192 high power laser beams in NIF will deliver 1.8 megajoules of energy and 500 terawatts of power onto a relatively small target.

Section 1 of this paper discusses the NIF cleanliness requirements by defining limits for molecular and particulate contamination to prevent laser-induced damage and reduced laser quality of the optical components. The requirements for the interior of the beampath are expressed in terms of less than Class 10 particulate levels and parts-per-billion AMC. Industrial standards that were current at the time were used to define surface level cleanliness with MIL-STD-1246C (superceded by IEST-STD1246D) and aerosol levels with FED-STD-209E (superceded by ISO 14644-1).

Section 2 discusses the CCP specifications for the interior of the NIF beampath and clean utilities. A graded CCP approach with different protocol levels and extensive training are used to achieve cleanliness requirements. The NIF beampath and utilities are being constructed by a partnership between LLNL and the construction contractor, JFI, in the stadium-size Class 100,000 building with localized mini-environments of Class 100 or better. Workers, including technicians, millwrights, ironworkers, electricians, carpenters, pipefitters, laborers, and engineers, are trained for different CCP levels and in the use of cleanroom garments, tools, and cleaning procedures.

Section 3 discusses several techniques for verifying beampath and utility system cleanliness: monitoring exposure, evaluating particulate levels with swipes, using the LLNL-developed PCVS, and measuring NVRs and AMCs with analytical chemistry techniques. Cleanliness measurements from the successful installation of one-quarter of the laser bay portion of the beampath indicate that the CCP specifications are achieving the NIF cleanliness requirements for the beampath and clean utilities.

\section{Section 1 - NIF Cleanliness Requirements}

NIF is concerned with both particulate and molecular contamination. Particulate contamination is common dust or dirt found in the air, on surfaces, or barely visible conglomerations of matter, such as metal flakes or cloth threads. Molecular contamination, on the other hand, is the cumulative buildup of individual molecules of foreign matter. Some molecular contaminants are in the air, which are referred to as AMCs, and some are on the surfaces in the form of NVRs. Cleanliness and contamination control in NIF is important to reduce the cost of optic refurbishment and to maintain laser performance.

Since NIF consists of more than 7,000 large optics, it is essential to extend their lifetime as long as possible. Contamination on the walls of vessels and enclosures can be dislodged and become contaminants on the glass laser slabs and other optical surfaces. Typically, small particles on surfaces are hard to dislodge because of Van der Waals and electrostatic forces. When scattered laser light strikes the walls, a thin surface layer of the wall expands rapidly which generates high inertial forces, and particles are dislodged from the walls to fall or be carried by thermal currents. If particulate or molecular contaminants land on NIF optics, the contaminants can absorb light, heat to very high temperatures, vaporize, and leave small craters on the surface of the optics, as shown in Figure 3. This damage can be caused by many different contaminants, including metal flakes, latex gloves, blue-jean lint, skin flakes, 
and road dust. Each damage site can scatter light out of the beam and lead to a small loss in laser energy and an increase in beam modulation.

Beamlet was a one-beam scientific prototype for the NIF laser that was operated at LLNL in the mid 1990s. At the completion of Beamlet operation, surface contamination levels defined in MIL-STD1246C were measured as shown in Figure 4. Even though the optics were installed at cleanliness levels that were lower than the surrounding beampath components, the optical and beampath surfaces appeared to achieve an "equilibrium" surface contamination level. Since the Beamlet levels were acceptable in terms of laser performance and damage mitigation, the NIF surface cleanliness requirements have been developed consistent with the measurements from Beamlet.

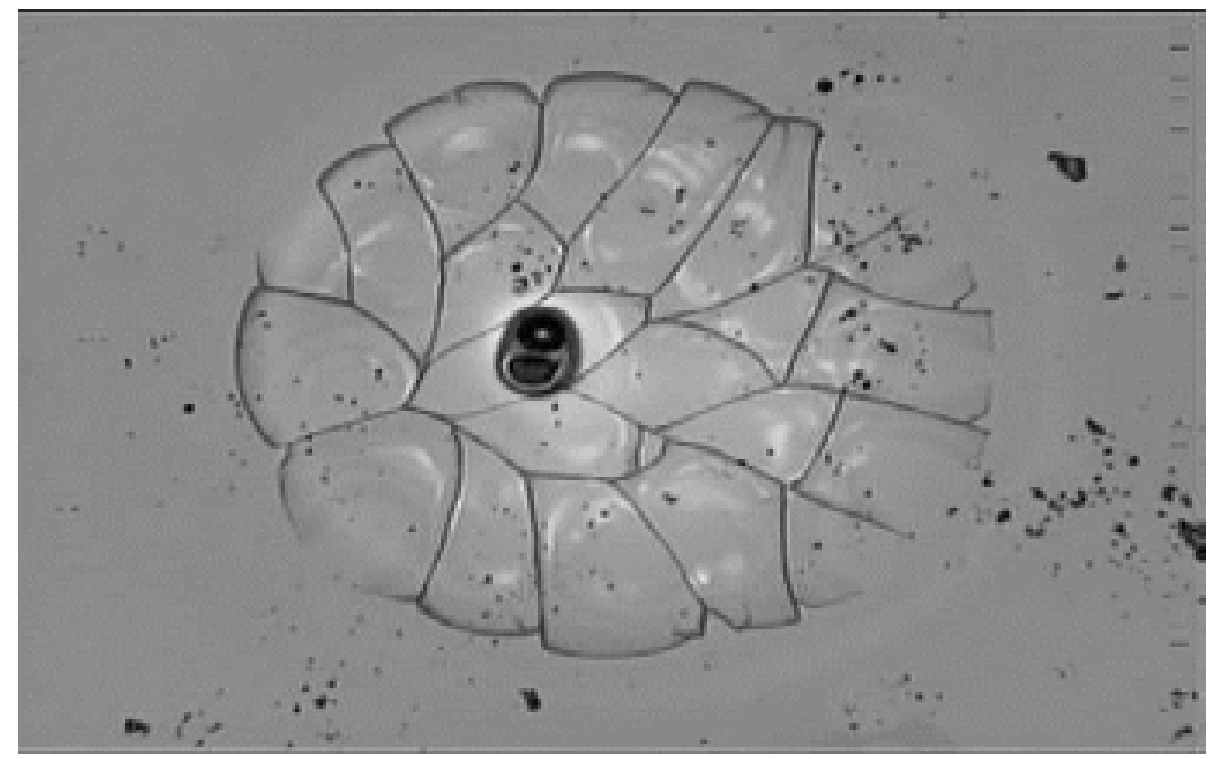

Figure 3. Result of contaminants deposited onto a laser slab and exposed to flashlamp firing.

Some of the optics, such as windows and lenses, are coated with low-density sol-gel antireflective coatings that are designed to enhance the amount of laser light that passes through the optic and to prevent scattered light off the optic. The low-density coating is very porous and spongelike and can adsorb AMCs. When the antireflective properties of the sol-gel coating are lessened, the amount of ghost reflections are increased and light is diverted from reaching the intended target. Small organic sources, such as motors, lubricants, and organic plasticizers in elastomer gaskets, can produce AMCs that change the refractive index, increase the reflectivity, and decrease transmission of sol-gel coated optics.

Current levels of AMCs in the Class 100,000 LTAB are quite low and will likely remain low because of the large volume of make-up air introduced into the air handling system that can flush AMCs from the air. It is important that materials containing significant levels of potential NVRs be carefully controlled both inside and outside of the beampath. Materials used in the beampath interior are either cleaned of NVRs on their surfaces or baked at high temperatures $\left(170^{\circ}\right.$ to $\left.200^{\circ} \mathrm{C}\right)$ to remove the NVRs. AMCs in the clean utility systems are controlled with point-of-use carbon filters. Although it is not possible to transport significant contaminants into the beam enclosure by vapor transport, contaminants with vapor pressures in the $10^{-3}$ to $10^{-7} \mathrm{~Pa}\left(7 \times 10^{-6}\right.$ to $7 \times 10^{-10}$ Torr $)$ range may be present in greases, adhesives, or other viscous liquids. Such materials have the potential to be transported by mechanical means, including handprints and garments, to internal enclosure surfaces. 


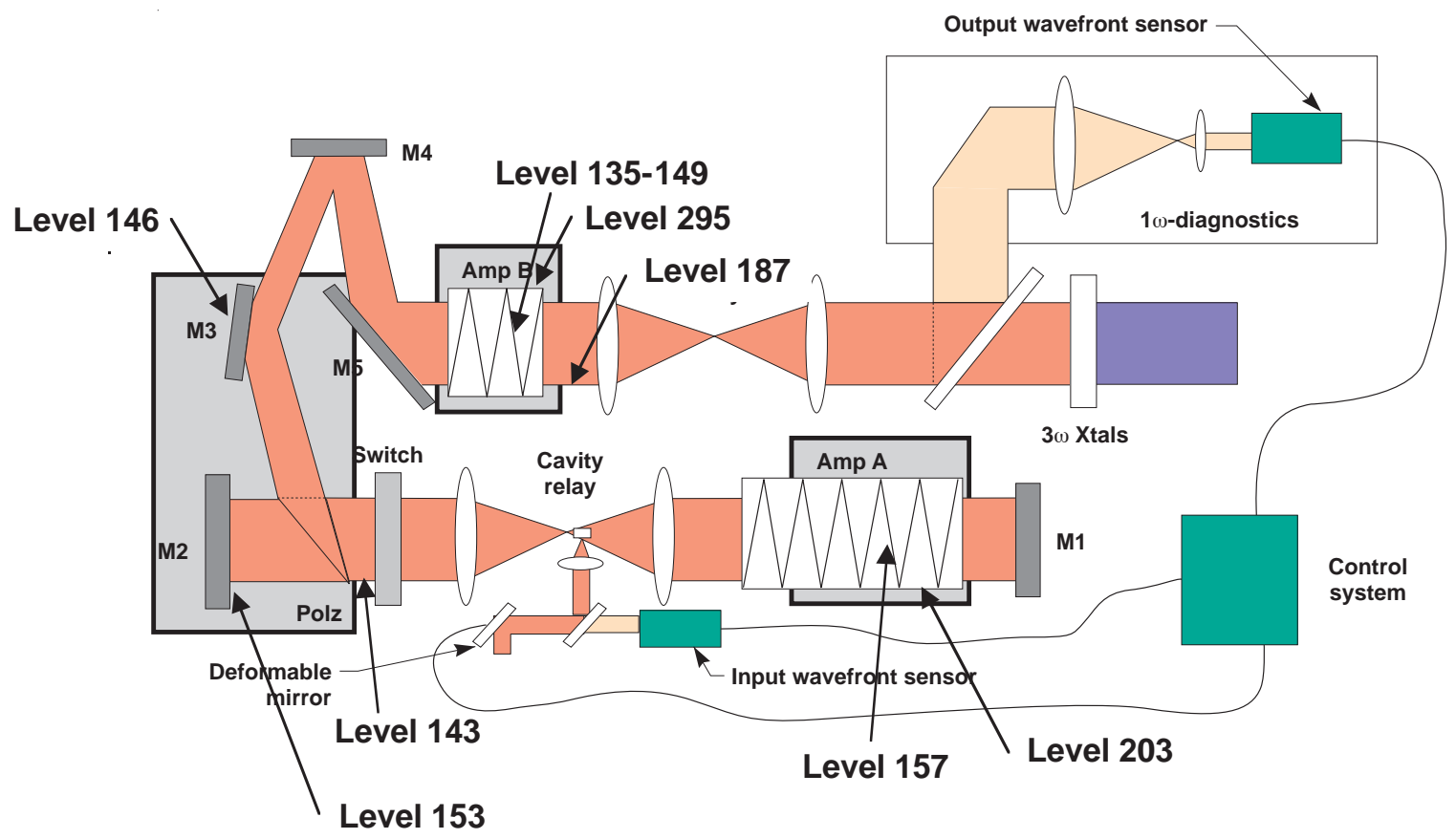

Figure 4. Surface cleanliness levels measured on Beamlet.

Organic contaminants in the molecular weight range of 250 to 500 grams per mole are of particular concern to sol-gel coated optics. Lower molecular weight organic solvents are relatively volatile and will easily evaporate from the walls of vacuum vessels and from sol-gel coated optics. However, higher molecular weight materials will only evaporate readily (and therefore transport and condense onto sol-gel coated optics) when in an environment in which the absolute pressure is similar to or lower than their characteristic vapor pressure. Since the NIF spatial filter components will operate at $10^{-5}$ Torr, an organic contaminant with a vapor pressure near this value is a potential outgassing source. The maximum allowable concentration of airborne molecular contaminants has been set to $20 \%$ of the vapor pressure of each individual contaminant, as shown in Figure 5. Since the characteristic vapor pressure of many organic compounds is closely related to their molecular weight, Figure 5 has the maximum allowable concentration, or $20 \%$ of the vapor pressure, as a function of the molecular weight.

Particulate and organic surface cleanliness requirements for beampath components are defined for NIF using the definitions in MIL-STD-1246C. Table 1 shows that the NIF maximum surface cleanliness requirements vary from their initial precision cleaning levels through commissioning of the beampath. Thirty years experience at LLNL with large solid-state lasers and with evaluating the effects of contamination on optics has established that NIF optical surfaces shall be cleaned to Level 50-A/10. To be consistent with these optical surface cleanliness requirements, the surrounding beampath components are precision cleaned to the levels shown in Table 1. These values coincide with the cleanliness levels that cleaning vendors can consistently achieve and are acceptable for both laser performance and rate refurbishment. The intent of these recommended values is to balance the investment in repeated initial cleaning cycles against the risk of degraded optical performance. Since the beampath is made up of large vessels, large-diameter pipe and duct, and small-bore tubing with a variety of materials such as rubber gaskets, sealants, and metals, the cleanliness levels for precision cleaning are achieved with several different cleaning techniques. These techniques include precision wiping, highpressure water wash, and ultrasonic cleaning. For removal of molecular contamination from nonmetallic components, precision wiping with isopropyl alcohol follows high-temperature bake-out. Small metallic components are washed in precision cleaning machines or dipped into ultrasonic baths. Large stainless steel components, such as vacuum vessels, are cleaned with a high-pressure, hot water wash 
that uses a surfactant to strip molecular contamination off the internal surfaces. The cleanliness requirements for the nonvacuum and vacuum portions of the beampath are slightly different because the optical surfaces in the vacuum portion are less sensitive to particulate contamination.

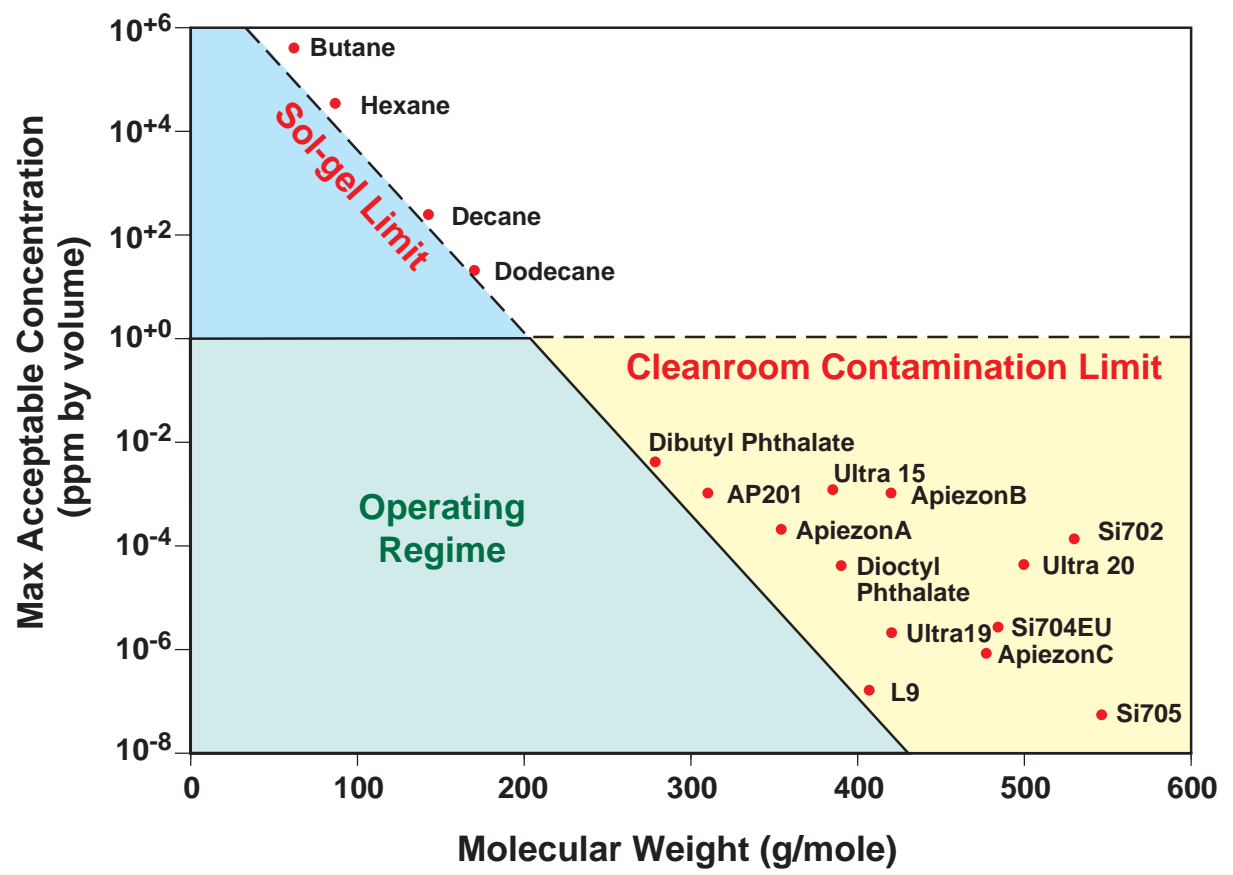

Figure 5. Concentration of organic contaminants to prevent degradation of sol-gel coatings.

Small differences between cleanliness requirements after cleaning and cleanliness requirements at the time of acceptance of the beampath components by the installation subcontractor, JFI, account for transport and storage effects that occur after precision cleaning. Following that acceptance, rigorous protocol procedures described in Section 2 are used during hardware installation to limit the particulate class-hour exposure and the introduction of additional organic contaminants. Cleanliness requirements for the completion of assembly and commissioning phases are shown in Table 1 . These requirements coincide with the turnover of the beampath from the installation subcontractor JFI to LLNL. To ensure that the assembly and commissioning phases have not compromised cleanliness levels, selective vessel entries and cleanliness measurements have been completed and are discussed in Section 3.

Table 1. Maximum surface cleanliness requirements (levels defined in MIL-STD-1246C).

\begin{tabular}{|l|c|c|c|}
\hline Region & $\begin{array}{c}\text { Completion of } \\
\text { Precision Cleaning }\end{array}$ & $\begin{array}{c}\text { Receipt of Beampath } \\
\text { Components by } \\
\text { Installation Subcontractor }\end{array}$ & $\begin{array}{c}\text { Completion of Clean } \\
\text { Assembly and } \\
\text { Commissioning }\end{array}$ \\
\hline $\begin{array}{l}\text { Laser Bay Beampath } \\
\text { (including amplifiers) }\end{array}$ & Level 100-A/10 & Level 120-A/10 & Level 140-A/3 \\
\hline $\begin{array}{l}\text { Switchyard and Target } \\
\text { Area Beampath }\end{array}$ & Level 100-A/10 & Level 120-A/10 & Level 140-A/3 \\
\hline $\begin{array}{l}\text { Laser Bay Vacuum } \\
\text { Beampath }\end{array}$ & Level 120-A/10 & Level 150-A/10 & Level 160-A/3 \\
\hline
\end{tabular}

The clean utility systems include piping up to 25 centimeters in diameter and sources for clean dry air (CDA) in the laser bays, argon in the laser bays and switchyards, CDA in the switchyards and target area, and vacuum in the laser bays and target area. The cleanliness requirements for these systems 
are summarized in the Table 2, and these systems provide the internal environment for the beampath. In Table 2, the requirements for gross-cleaned piping and ducting or regions upstream of the combination carbon and HEPA (ULPA) filters, are differentiated from those for precision-cleaned piping. Downstream of the combination filters, the precision-cleaned piping has bake-out requirements for nonmetallic components. The cleanliness requirements are listed in terms of constituents in particulate or organic concentrations and are consistent with physics or performance considerations for the different regions of the beampath. In general, the physics requirements for the gross cleaned regions are less stringent than for the precision cleaned regions and the differences are based on filter efficiencies between $99 \%$ and $99.999 \%$ depending on the constituent.

Since the piping and large ducting for the clean utility systems will be installed to specific interfaces, the installation requirements for the systems are defined at those interfaces. The requirements for the supply are satisfied for either CDA or argon with appropriate filtering and processing. Since the constituents are controlled at the supply, not all of them are measured throughout the rest of the clean utility systems. Instead, several representative constituents, dibutyl phthalate (DBP) and hydrogen sulfide $\left(\mathrm{H}_{2} \mathrm{~S}\right)$, are monitored in the clean utility systems. This approach is consistent with the specifications for the combination carbon and HEPA (ULPA) filters that separate the gross and precision clean sections by using industry practice to represent the multiple compounds with an equivalent compound that can be specified in a test. For carbon filters, the factory testing requirements for filter efficiencies are specified in terms of toluene and sulfur dioxide as the test gases. The only cleanliness requirement that will be measured in the gross clean section is the particulate class since this section is upstream of the carbon filters. For the precision clean piping and ducting, the requirements are provided at the outlet from the precision clean section and downstream of the combination carbon and HEPA (ULPA) filters.

The clean utility requirements attempt to accommodate industry practice and account for the self-cleaning aspects of the utility systems during a flow of clean gas in the piping. Specifically, the recirculation feature of the CDA system and the pump-down characteristics of the vacuum system will contribute to decreasing the particulate and organic concentrations in the piping. In addition, the vacuum roughing lines do not have HEPA or carbon filters since there is low risk of back streaming through the roughing pumps. On the other hand, the vacuum back-fill lines will have HEPA and carbon filters and will meet the precision cleanliness requirements.

To achieve the cleanliness requirements in Table 2 , the recommended techniques include purchasing clean pipe and "oxygen-service" cleaned instrumentation, precision wiping, high-pressure water and surfactant washing, and ultrasonically cleaning. The goal is to have a consistent and implementable set of requirements for the appropriate beampath utility systems and to use industryaccepted practices. While the clean utility systems have not been installed yet, several samples of clean pipe have been fabricated, and their cleanliness levels are consistent with the requirements in Table 2.

Table 2. Cleanliness requirements for the clean utility systems.

\begin{tabular}{|l|c|c|c|}
\hline \multicolumn{1}{|c|}{ Constituent(s) } & Supply (Source) & $\begin{array}{c}\text { Gross Clean Piping and } \\
\text { Ducting }\end{array}$ & $\begin{array}{c}\text { Precision Clean Piping } \\
\text { and Ducting }\end{array}$ \\
\hline Airborne particulate & Class 100 & Class 1,000 & Class 1 \\
\hline Total hydrocarbons & $0.10 \mathrm{ppm}$ (volume) & not measured & not measured \\
\hline Organics & $0.01 \mathrm{ppm}$ (volume) & not measured & not measured \\
\hline Dibutyl phthalate & not measured & not measured & $1 \mathrm{ppb}$ (volume) \\
\hline Hydrogen sulfide gas & not measured & not measured & $1 \mathrm{ppb}$ (volume) \\
\hline Sulfur & $0.01 \mathrm{ppm}$ (volume) & not measured & not measured \\
\hline Halogens & $0.01 \mathrm{ppm}$ (volume) & not measured & not measured \\
\hline Amines, imines, nitrogen & $0.01 \mathrm{ppm}$ (volume) & not measured & not measured \\
\hline Carbon dioxide & not measured & not measured & not measured \\
\hline
\end{tabular}




\section{Section 2 - Clean Construction Protocol (CCP) Specifications}

The precision-cleaned surfaces of the beam enclosures are only exposed to the LTAB environment for short periods of time as necessary to perform the clean assembly. Since a limited amount of contamination could occur during clean assembly, the CCP procedures were developed to track exposure time and aerosol conditions to limit the exposure in terms of class-hours. To minimize and monitor the contamination levels during assembly, the "exposure," or class-time product, for the interior of the beampath components, is specified. Exposure is related to the settling of aerosols from a specified environment, or aerosol class, over a specified period of time, which is usually measured in hours. A higher value of exposure results from either a higher class of aerosol or a longer period of time the surface is exposed to the room air. For beampath components, the total allowable exposure during assembly is specified as 20,000 class-hours, and since several different activities occur during assembly, Table 3 shows that the 20,000 class-hours are divided into four allocations of 5,000 class hours each. For ambient conditions between Class 1,000 and Class 5,000, each step of the clean assembly can be completed over a period of one to five hours. If longer periods of time are necessary to complete a complex assembly, local cleanrooms can be established to provide Class 100 or better aerosol conditions. The intent of the CCP specification is to achieve less than 20,000 class-hour exposure by limiting the exposure of each clean activity.

There are several challenges associated with clean beampath installation including the large number of connections, mechanical fit-up considerations, safety issues, the cleanliness of the LTAB, the "supply train" of beampath components, and the integrated schedule of construction activities. There are approximately 4,500 clean connections required for NIF and these connections must be made in space and schedule constrained conditions within a nominally Class 100,000 environment.

Table 3. Precision cleanliness exposure requirements.

\begin{tabular}{|l|l|}
\hline Assembly Process & Exposure Limit \\
\hline Subassembly in a Clean Room & 5000 Class-hours \\
\hline Clean Connection of Beampath Components & 5000 Class-hours \\
\hline Access Interior of Beampath & 5000 Class-hours \\
\hline Connection of Utilities to Beampath & 5000 Class-hours \\
\hline
\end{tabular}

The requirements for the clean assembly of the beampath and utilities are provided in Construction Subcontract Packages (CSPs) that were developed by LLNL for the contract with JFI. Three methods are defined in the CSPs for installing precision-cleaned components in clean work zones: (1) the use of portable cleanrooms within the LTAB, (2) the use of "fast connections," and (3) the use of pressurized connections in which HEPA filtered air is forced through the clean beampath and exhausted into the LTAB. Portable cleanrooms are Class 100 to 500 enclosures that permit additional time for clean assembly efforts and resolution of assembly issues since the 5,000 Class-hour limit results in 10 to 50 hours of exposure. A limit of 10 hours for the exposure of a precision cleaned surface in a portable cleanroom is established to mitigate unnecessary exposure.

For the "fast connection" method shown in Figure 6, local air quality is a maximum of Class 5,000 in a defined space ten feet from the intended clean connection in all horizontal directions and extending from the floor to the ceiling. Exposure time is one to five hours depending on the air quality as defined by the 5,000 Class-hour limit. In contrast to using a portable cleanroom, the "fast connection" relies on the ambient conditions of the LTAB. This technique is used for locations with confined space that precludes the use of a portable cleanroom or schedule constraints for fabricating a portable 
cleanroom. In Figure 6, Joints A, B, and C are all considered within the zone for "fast connection." No particle and aerosol-producing activities, such as grinding, welding, painting, or sanding, is permitted within 25 feet of the clean work zone during a clean connection.

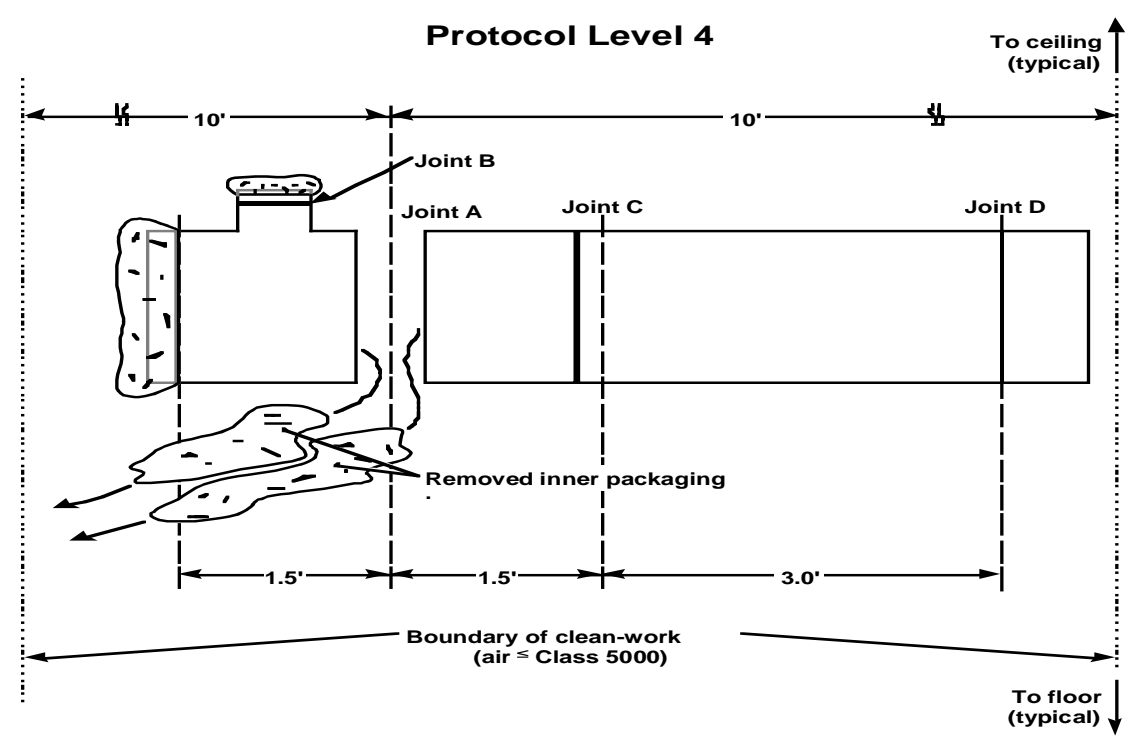

Figure 6. Fast connection method.

For a pressurized connection, as shown in Figure 7, a Class 100 clean air supply is attached by umbilical hose (elephant trunk) through an opening at least two feet away from the opening of the intended connection. The umbilical hose is sealed into the opening by using ultra clean polyethylene film and cleanroom tape. To minimize the effects of vortices near the opening, the HEPA filtered clean air supply has a static pressure of $25 \mathrm{~Pa}$ and a minimum free flow of $60 \mathrm{cfm}$ per square foot of enclosure cross-section. Computational fluid dynamics models were constructed, such as the one shown in Figure 8 , to predict the volumetric flow rate needed to prevent vortices from bringing less clean air into the opening. With sufficient velocity of clean air, the flow is maintained such that the air always goes from the clean component into the ambient environment. For the switchyard enclosures in Figure 8, the flow at the bottom opening prevents exterior air from entering at the top opening and at the side of the bottom opening near the closed end.

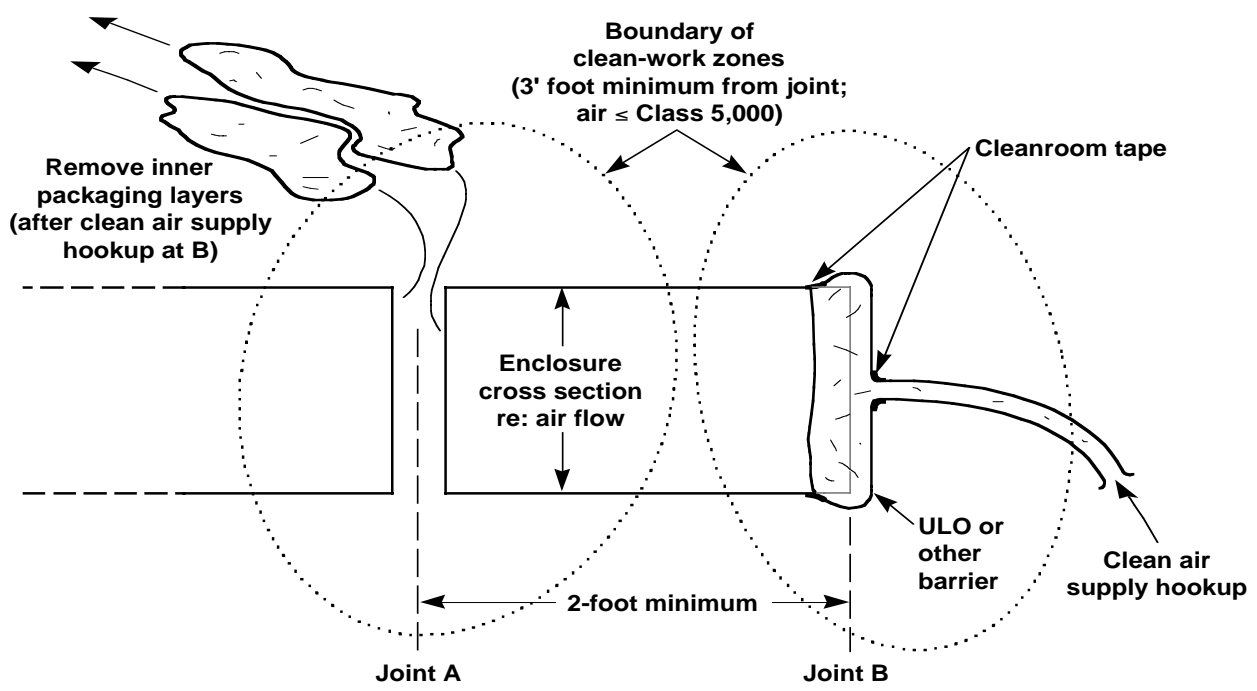

Figure 7. Pressurized connection. 
To implement the three connection techniques discussed above, the CSPs have a graded approach for CCP levels and all activities performed within a protocol level must conform to the rules of that level. The protocol level will vary between Level 2 and Level 4 as work varies between installing beampath components and less contamination-critical components.

Protocol Level 1 is the lowest level, and there are no access restrictions associated with Level 1. Prior to reaching Level 1, all clean construction personnel attend CCP training. Work performed at Level 1 is heavy construction, such as rigging large components, welding, and the removal of the outside layer of protective packaging from precision cleaned equipment.

Protocol Level 2 is initiated after installation of the systems that distribute clean air to the LTAB and the establishment of a means of a positive pressure within the LTAB. Prior to initiation of Protocol Level 2, the LTAB structures, walls, and floors are washed, wiped, or vacuumed to a visibly clean condition. Rudimentary airlocks are installed at all LTAB access points. Before entering Protocol Level 2, employees must pass through a temporary gowning facility that is housed within the airlock and where clothing, tools, and materials may be cleaned. Continual cleanup is required for Protocol Level 2, and all material used in Level 2 must be standard clean. While gowning is not required, hairnets and shoe covers are worn and no food or drink is permitted. All welding, drilling, cutting, and grinding operations have local means of debris removal, such as cleanroom vacuums.

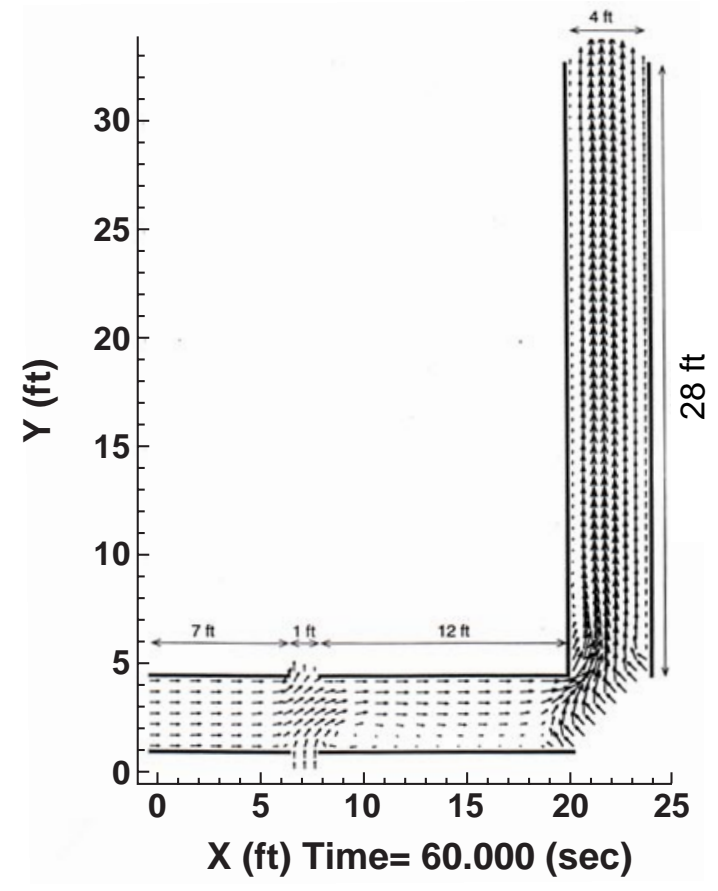

Figure 8. Analytical predictions of clean air flow patterns with pressurized connection.

Protocol Level 4 occurs for clean work zones in which precision cleaned surfaces are exposed to the LTAB or portable cleanroom environment. Personnel working at this level receive additional training and those directly responsible for installation of beampath components are certified to perform those activities as defined in the CSPs. The access points to Protocol Level 4 areas are through protocol Level 2 areas and Level 4 access is limited to one primary entry and exit point for personnel with required log-in and log-out controls. Work performed at this level includes installation, connection, alignment, and pressurization of all beampath components. This includes all work where the interior of any of the beampath components is exposed to the LTAB environment. For Level 4, the Level 2 rules are enhanced by requiring cleanroom gowning with gloves, coverall, booties, hoods, and facemasks. 
Tools and equipment used near clean connections are precision clean, and no debris generating activities are permitted. For access into precision clean components, enhanced Level 4 gowning requirements are implemented with full-body and cleanroom suits that can be laundered, with breathing through a cleanroom helmet and shield system.

The implementation of the CCP program relies on extensive training, the involvement of contractor field engineering, and the solicitation of feedback from the workers. The goal is for the laborforce of tradespeople, engineers, and managers to buy-in or accept the CCP program so that individuals feel personally responsible for the CCP activities. With so many CCP activities occurring simultaneously there is no cost effective technique to have independent monitors at all places and at all times. Instead, each individual worker is expected to be their own monitor with the CCP program providing the framework for and independent assessment of each individual's efforts.

Implementation of the CCP program on NIF starts with five phases of training. Phase One is a four-hour overview of all aspects of CCP that focuses on NIF cleanliness requirements. All of the construction management team members and subcontractor supervisory personnel must attend to learn the aspects of CCP from a management perspective. It has been found in previous CCP programs that failures occur when management-level personnel are not thoroughly trained and aware of CCP considerations.

Phase Two of CCP training is required for all personnel involved in clean construction activities in the NIF building and must be completed prior to access into the facility. This one-hour course covers the rules for Protocol Levels 1 and 2 and describes the purpose of the NIF facility in general terms. Phase Three covers general cleaning and clean work zone requirements and the rules for Protocol Levels 1, 2 and 4. This four-hour phase of training is necessary for all hands-on construction personnel working within the cleanroom or clean work zones during Protocol Level 4 and for the QA/QC personnel monitoring those activities. Phase Three training is interactive and includes demonstrations of gowning, wipedown, and cleaning procedures. Real life examples are an important part of the training at all phases. Phase Four training is for CCP personnel supporting and monitoring efforts in Protocol Level 4. This four-hour session covers instrumentation and quality control sampling procedures discussed in Section 3, such as particle counters, particle swipes, and NVR measurements. In the class, swipes are performed on relatively dirty and clean surfaces to gain practical experience and using the PCVS to evaluate the swipes.

Phase Five training is an intensive, eight-hour session for hands-on construction personnel who will perform clean connections or work within the confines of precision cleaned vessels. This training includes gowning for Level 4, performing clean connections, precision wiping, and entering precision cleaned enclosures. For cleanroom gowning, a common mistake is to drag the legs of the coverall on the floor while putting them on which essentially wipes the floor with the "clean" coverall. Instead, personnel are taught to bunch up the legs of the coverall and slip their feet through and then simply roll down the pant legs. The training also provides an overview of the quality control sampling procedures covered in Phase Four training. The clean connection crews are the primary personnel maintaining the strict cleanliness specifications for NIF. Since the NIF training may be the first time many are exposed to CCP, the impact of cleanliness on the performance of NIF is demonstrated. At the end of a class, the instructor passes around cleanroom gloves for the students to inspect. Typically, no one notices anything unusual until the instructor places air into the glove, and it becomes obvious that a tiny piece of cloth is missing from the thumb of the glove. If this small piece of cloth had fallen on the laser glass, it could have caused the glass to crack.

NIF CCP training encourages honesty and integrity when reporting progress, results, and nonconformance. Since only a relatively small portion of the beampath will have cleanliness measurements performed on it, the CCP program relies on trends and self-monitoring. As an example of self-monitoring, a NIF installer left the interior of a precision-cleaned vessel and realized a piece of 
glove was missing. This was immediately reported to the supervisor, and the operation was stopped until the piece of glove was found. The installers were praised for taking responsibility to achieve the CCP. NIF CCP training encourages feedback and suggestions from the participants, especially the personnel performing the process, because improvements in efficiency, safety, and CCP will come from these personnel. Constant communication involves field engineers walking the construction site regularly and talking with the workers to ask about the status of the processes and how the CCP is working. Thorough training efforts and effective enforcement policies are important, but the key to success is when the workers accept individual responsibility for the CCP.

\section{Section 3 - Verifying CCP Specifications}

The CCP program discussed in Section 2 has been tested and implemented during the clean installation efforts associated with "fast connection" tests conducted in AmpLab in September 1999, the first vessel entry in March 2001, and completion of the Cluster 3 beampath in October 2001. Results and experiences from these efforts have been evaluated to upgrade the $\mathrm{CCP}$ specifications and to correlate airborne and surface contamination levels.

In 1997, LLNL researchers realized that neither the aerospace nor the microelectronic fabrication industries had a means of evaluating surface particulate cleanliness that satisfied NIF requirements, so the Particle Cleanliness Validation System (PCVS) was developed as a filter paper swipe, or "white glove" technique. PCVS is a combination of a surface particle collection tool and a microscope based data reduction system for determining the particle cleanliness of mechanical and optical surfaces. Using this technique, particles are collected on membrane filter paper that is swiped on a surface for a specified distance to collect sufficient particles to significantly exceed the typical cleanliness of the filter paper. The swipe paper is then placed into a cassette for protection from further contamination and transported to a charge-coupled device camera-equipped microscope with motorized stage and image analysis software that counts the particles.

To satisfy NIF requirements, the analytical procedure to measure NVRs involves three steps. The first step is to rinse the test surface with solvent and completely capture it in the sampling container. Next, the solvent is concentrated to near dryness by evaporating the solvent first by bubbling with a clean gas, such as helium or nitrogen, and followed by blow-down. The residual solvent with the NVR is transferred into progressively smaller containers to minimize the container surface area and potential loss of NVR. Finally, the third step is to transfer the remaining solvent containing the NVR into a weighing boat and to weigh the dry residue to determine the NVR. At this point, the samples can be analyzed by a gas chromatograph mass spectrometer if information concerning the identity of the contaminants is required.

AmpLab was built at LLNL in 1995 to demonstrate the feasibility of the NIF amplifier configuration by testing amplifier components and validating amplifier performance prediction codes. AmpLab used full-size NIF components to demonstrate the clean assembly and operation of the amplifiers and the feasibility of the bottom-loading and clean-mating techniques used throughout NIF to install and assemble components. The clean assembly techniques from AmpLab were the basis for the development of the CCP specifications for the beampath installation. In 1999, four clean assembly tests were performed in AmpLab with a range of garment requirements and installation sequences. The tests involved working with full-size enclosures, working in space-constrained conditions, and using CCP rules developed for the clean installation of the NIF beampath. The tests showed that the entire connection sequence for a NIF enclosure, which is approximately 1.2 meters by two meters in plan dimensions, could be completed in less than forty minutes and the surface cleanliness could be

maintained by limiting the exposure. Several different types of cleanroom garments and two different 
installation techniques were used in the AmpLab tests, which involved using a lift to install an approximately one-meter-long enclosure and bellows subassembly between previously installed beampath components. One technique involved removing the protective covers from the precisioncleaned components prior to raising the enclosure subassembly into the beampath, while the other technique involved removing the covers as a guillotine with the enclosure subassembly between the previously installed beampath components. The space for installing the enclosure subassembly was created by compressing the bellows.

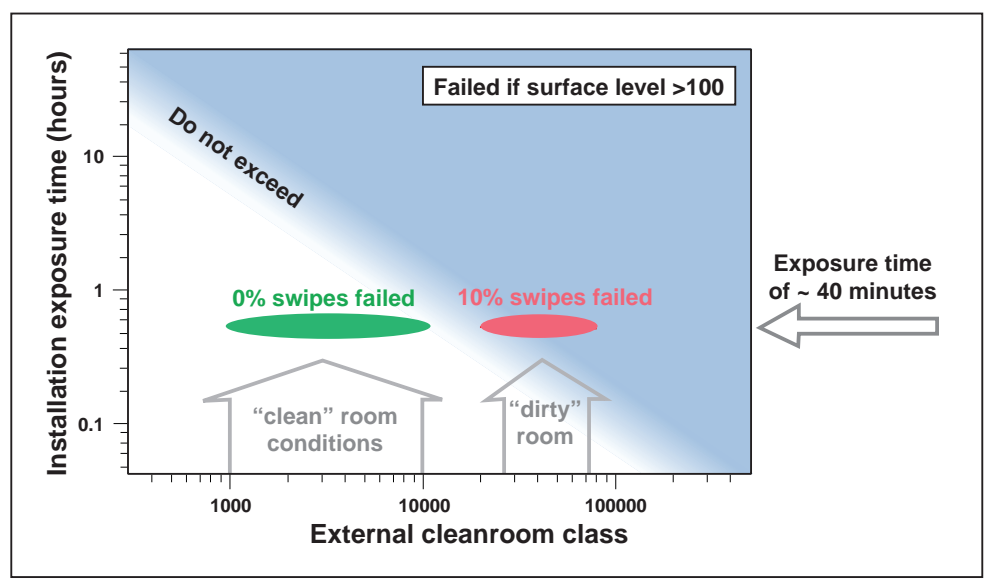

Figure 9. Results of clean connection tests in AmpLab.

While the garments and installation sequence had some influence on the surface cleanliness, Figure 9 shows that the ambient conditions, or exposure, had the biggest impact. The air class was altered in AmpLab by changing the flow rate of the air through the room HEPA filters. For clean assembly in Class 10,000 or below conditions, all the PCVS swipe results were below Level 100. About ten swipes were made on the bottom surfaces and walls of the installed components by removing a cover on the bottom of the installed components. For clean assembly in about Class 20,000 conditions, some of the swipes exceeded Level 100. Since the major source of particulate contamination during clean assembly is people, the results in Figure 9 show that the different garments and cover removal techniques had similar influence. As long as cleanroom protocol is used to mitigate the influence of people, the surface contamination levels are limited by controlling exposure.

The CCP for efforts inside a precision-cleaned component were evaluated during the first vessel entry in early 2001. The cavity and transport spatial filters each have three relatively large vacuum vessels that are fabricated from stainless steel and divided into six bundles. Two of the vessels are end vessels that are approximately 9.3 meters wide, 3.5 meters long, and 2.5 meters tall. Cavity Spatial Filter end vessel 2 (CSF-2) was precision cleaned to Level 83-A/10 using a high-pressure water spray technique and packaged for storage. In mid-2000, CSF-2 was taken out of storage so precision-cleaned vacuum covers could be installed over the openings on one side. In the course of this clean installation, general rouge, or light rust, was observed on the floors of two of the six bundles. In early 2001, the inspection of the interior of the vessel and in-situ cleaning of lightly rusted areas was performed.

To enter CSF-2, a temporary clean enclosure was erected under the entire length of the vessel. Supported on a scaffold platform, this clean enclosure was constructed from ultraclean polyethylene sheeting and provided individual "rooms" beneath each bottom port. There was also a separate gowning room at the entrance. Clean entry to each bundle of CSF-2 was through a $0.6 \mathrm{~m}(2 \mathrm{ft})$ diameter port cover on the bottom of the vessel. Since people had to enter and work in the precision-clean vessel, enhanced CCP Level 4 requirements were used to minimize the potential contamination from people. Finally, all surfaces that were touched during the interior vessel effort were wiped clean. 
To evaluate the cleanliness of the vessel interior, especially the rouge, a swipe map and table were developed. The swipe map and table identified the locations of interest and the type of swipes to be performed. For beampath installation, swipe maps and tables are developed for the interior surfaces of all the precision-cleaned components. An important feature of the vessel swipe tables is the requirement of entry and exit swipes to track the impact of people working inside the vessel. If the exit swipes do not meet the surface cleanliness requirements, then further wiping is performed on the interior surfaces. For CSF-2, it was discovered that the vessel was sufficiently clean following wiping and that PCVS inspection of the swipes was sufficient to decide whether to further clean a surface or not. To perform an in-situ cleaning of the vessel, a 10\% IPA or dry wipe of surfaces was a safe and effective method to reduce the particulate contamination on stainless steel surfaces to values below the limits in Table 1.

In addition to demonstrating the effectiveness of wiping to clean previously cleaned surfaces and of the PCVS to measure cleanliness levels, the efforts in CSF-2 provided successful experience with exposure monitoring. Figure 10 shows the particle counts in the clean enclosure below the vessel, and the effects of work activity are illustrated. Without activity in the clean enclosure or vessel, the particle counts are below Class 10. The counts approach Class 500 when people enter the clean work zone and spikes occur either due to a large amount of activity or people in the clean work zone. With the implementation of the Level 4 protocol specifications, the 5,000 Class-hour exposure limit was not exceeded and the cleanliness conditions were maintained.

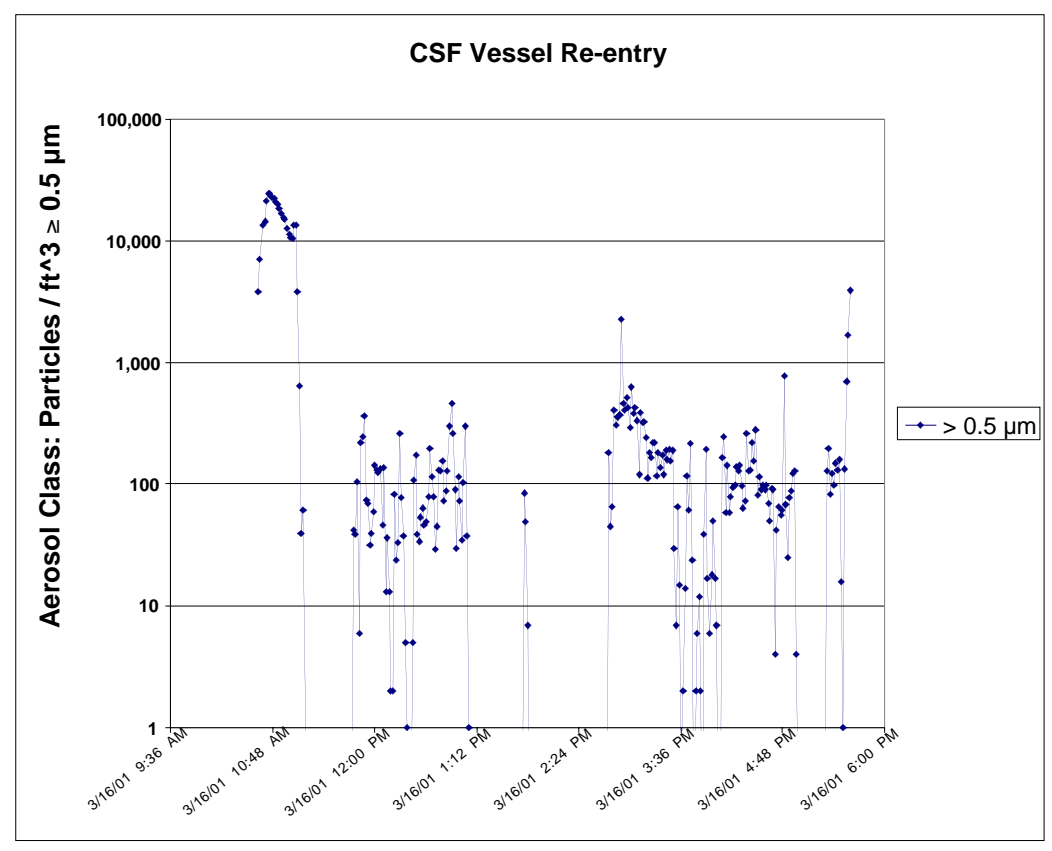

Figure 10. Particle concentration in enclosure air as a function of time for entry into vessel.

In addition to using the PCVS and particle counts to verify cleanliness, NVR measurements were performed on the manport flanges. Before vessel entry, the NVR values ranged from 0.036 to 0.079 $\mathrm{mg} / \mathrm{ft}^{2}$ and were below Level A/10. Following clean efforts in the vessel, the NVR values ranged from 0.086 to $0.115 \mathrm{mg} / \mathrm{ft}^{2}$ and were measurably and consistently higher in each bay after entry. Since the NVR levels were indistinguishable from Level A/10, it was concluded that clean work zones were effective to limit both particulate and NVR levels. Since the CSF-2 gas supply for the vessel interior did not have carbon filtration, and a slight increase in NVR levels was measured, a combination of HEPA and carbon filtration is required for gas directly inserted into the beampath. 
With the results and experiences from the AmpLab tests and first vessel entry, the CCP requirements discussed in Section 2 were fully implemented for the assembly and installation of the Cluster 3 beampath during late 2001. In each laser bay, there are two clusters of beamlines for a total of four NIF clusters. Cluster 3 was the first set of beampath components precision aligned, cleanly installed, and tested. As shown in Figure 11, Cluster 3 contains two amplifiers, two spatial filters, two mirror support structures, and over 100 connecting components between the amplifiers, spatial filters, and support structures. In general, the cluster-wide spatial filter vessels and structures were precisioncleaned to the levels in Table 1 and then were installed and precision-aligned. During installation of the components in the Laser Bay during Protocol Level 2, the interior surfaces were protected with packaging consisting of an intimate layer of ultra clean polyethylene and a protective barrier of hard plastic (as shown in Figure 11 for Cluster 4 components) or a metal cover sealed with a gasket. Following installation of the vessels and structures, the connecting components were installed in four different types of clean work zones. These clean work zones implemented Protocol Level 4 requirements and in some cases, workers had to enter the precision cleaned components.

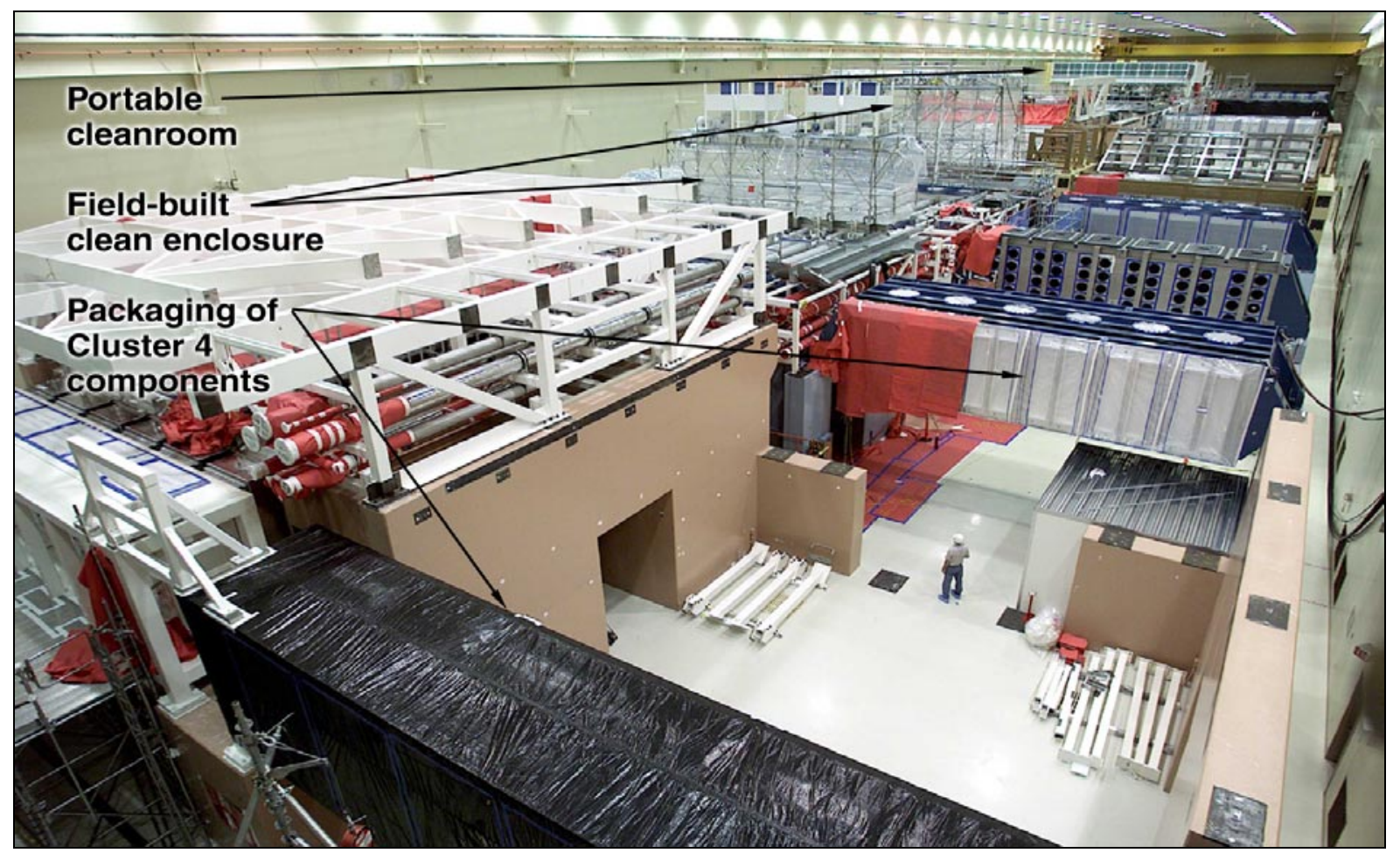

Figure 11. Installation of NIF Cluster 3 with Cluster 4 shown on the right.

For clean installation of connecting components, the four types of clean work zones were: portable cleanrooms, field-built clean enclosures, "fast connections," and pressurized connections. The portable cleanrooms were certified Class 100 or better cleanrooms that ranged in size from 10 by 10 foot to 30 by 30 foot. In Figure 11, the largest of the portable cleanrooms is shown positioned at the far end of Cluster 3 for both clean installation of connecting components and access into a spatial filter vessel. The portable cleanrooms were positioned so they did not have to be moved often and could accommodate lifts for people and components during clean subassembly of beampath components. To accommodate field conditions and to have flexibility for locations of clean work zones, field-built clean enclosures were fabricated with scaffolding and fan-filter units (FFUs), as shown in Figure 11. These clean enclosures were a novel approach developed by JFI to combine the benefits of clean air from 
FFUs with the construction flexibility of scaffolding for work platforms. As Figure 11 shows, the beampath is located about 4.5 meters above the ground and access to the beampath can be constrained. For a clean work zone, the scaffolding was wiped clean, wrapped in ultraclean polyethylene, and the FFUs were typically attached to the scaffolding "roof." Depending on the size of the clean enclosure, two different types of FFUs were used and they included the standard 2- by 4-foot HEPA filter units used in cleanrooms and larger 4- by 4-foot blowers that produced about 1,400 cfm of carbon and HEPA filtered air. The larger units were also used to provide filtered air for efforts inside the clean beampath, such as installation of kinematic mounts in the spatial filter vessels. Prior to installation efforts in the clean work zones developed by portable cleanrooms or field-built enclosures, the area was wiped and the clean air was verified to be Class 500 or better.

At several locations, the access or schedule constraints prevented the use of portable cleanrooms or field-built clean enclosures. The "fast connection" technique, as shown in Figure 12, was used at these locations. In order to use the "fast connection" technique, the ambient aerosol conditions were maintained below Class 5,000, and the installation time was limited to an hour. In addition, the clean work zone was wiped down prior to the installation, and Protocol Level 4 requirements were implemented for the workers. In Figure 12, the workers are following Protocol Level 4 requirements and the beampath components have the ultraclean polyethylene covers attached by cleanroom tape in preparation for removal. The safety harness and hat are either wiped clean and worn outside the cleanroom suit or are worn underneath the cleanroom suit with slits in the cleanroom suit for safety equipment.

As defined by swipe maps and tables, the surface cleanliness levels were measured during different stages of the Cluster 3 installation. In general, receipt swipes were performed on all the beampath components and compared to the receipt swipe requirements in Table 1. Depending on access constraints, a limited number of additional swipes were performed following subassembly or installation, which were also compared with swipe requirements. The swipes were performed near the open ends of the beampath components within a clean work zone. If the surface cleanliness levels exceeded the requirements either during receipt or following installation, the surfaces were precision wiped and reswiped. The swipe results for Cluster 3 spatial filter beamtubes, interstage enclosures, and vacuum vessels are shown in Figure 13. These results include both receipt and post-installation swipes, and they are generally below the requirements. The swipe results above Level 200 were receipt swipes and these components were wiped and re-swiped prior to installation. Less than $10 \%$ of the final, or exit, swipes for Cluster 3 exceeded the requirements, and in those cases, it was determined that the contamination risk to either re-enter the beampath or remove the component exceeded the benefit of rigorously satisfying the swipe requirement. NVR measurements were also performed in several locations of the installed beampath, and all these measurements were below Level A/3. In general, the implementation of the CCP requirements was successful in achieving acceptable surface particulate and NVR levels.

In addition to particulate and NVR measurements, the cleanliness exposure, or class-hours, for the clean installation activities was tracked. The class-hours for the installation of interstage enclosures using both a field-built enclosure and the "fast connection" technique are shown in Figure 14. The maximum value for the field-built enclosure is less than Class 250 and the nominal value is about Class 100. For the "fast connection," the maximum value approached Class 3,500, while the nominal value for ambient conditions was about Class 1,500. For both types of clean work zones, the time to install the beampath components ranged from about 30 minutes to almost 2 hours. In general, the cleanliness exposure for subassembly and installation efforts was much less than the 5,000 class-hour requirement. While the CCP program and Protocol Level 4 requirements mitigated particulate contamination from people, the exposure limits minimized both particulate and molecular contamination from the environment. 


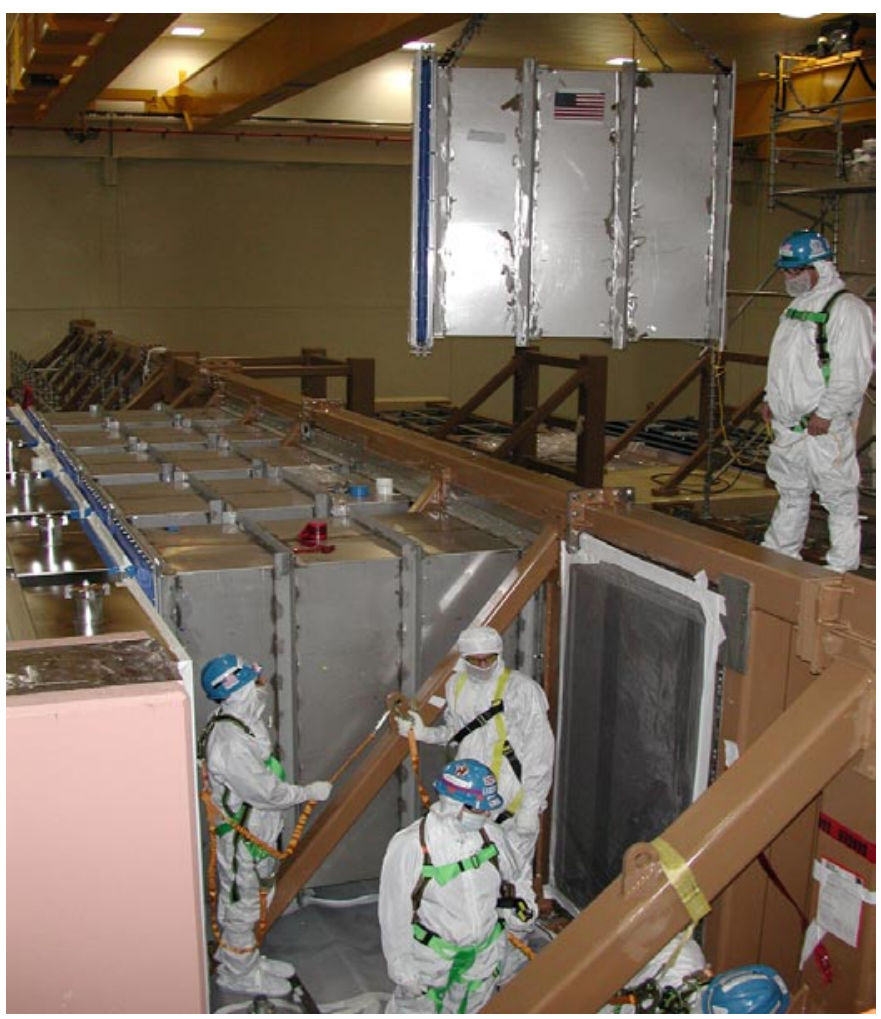

Figure 12. Fast connection of interstage enclosure.

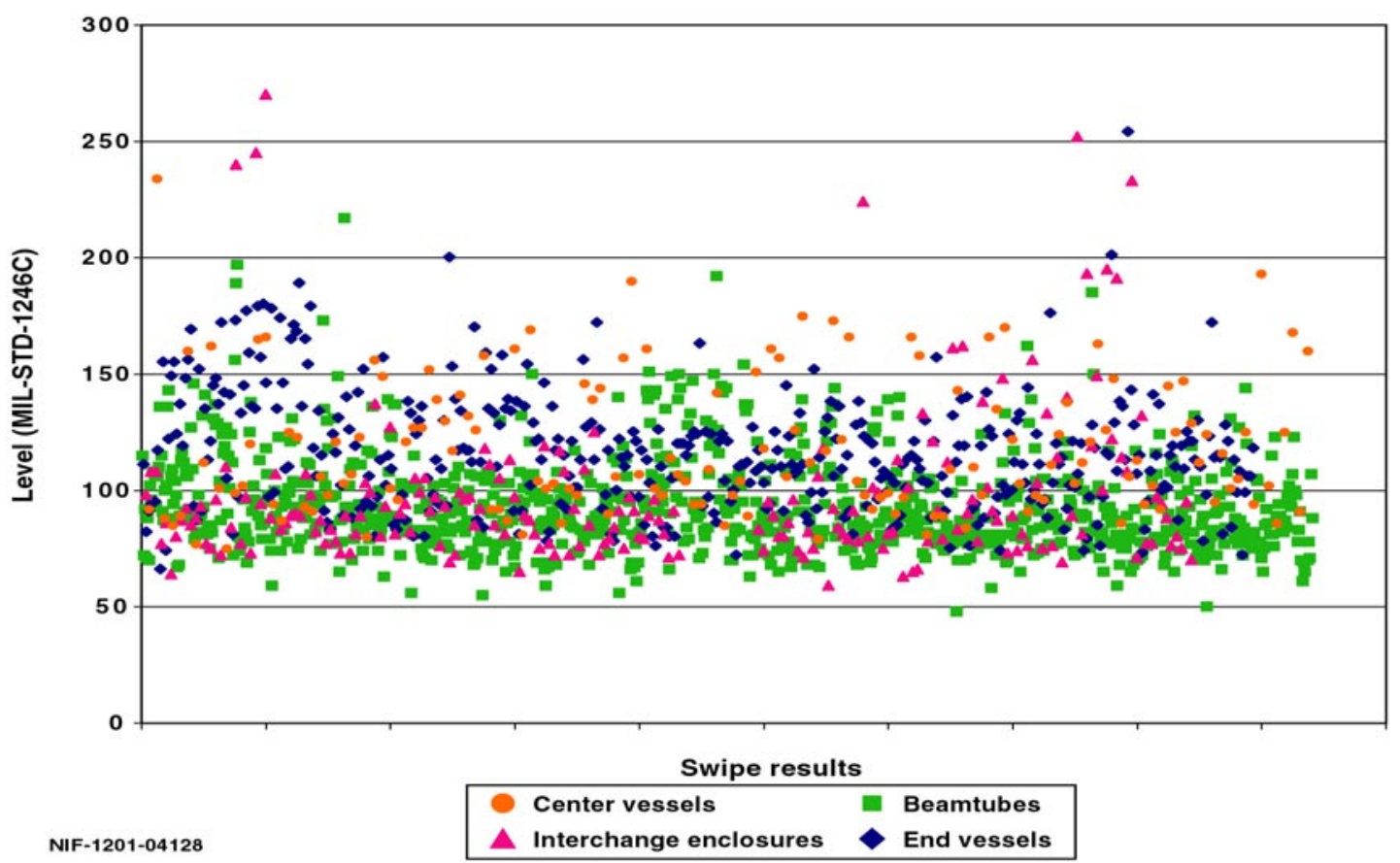

Figure 13. Swipe results for the vessels, interchange enclosures, and beam tubes. 


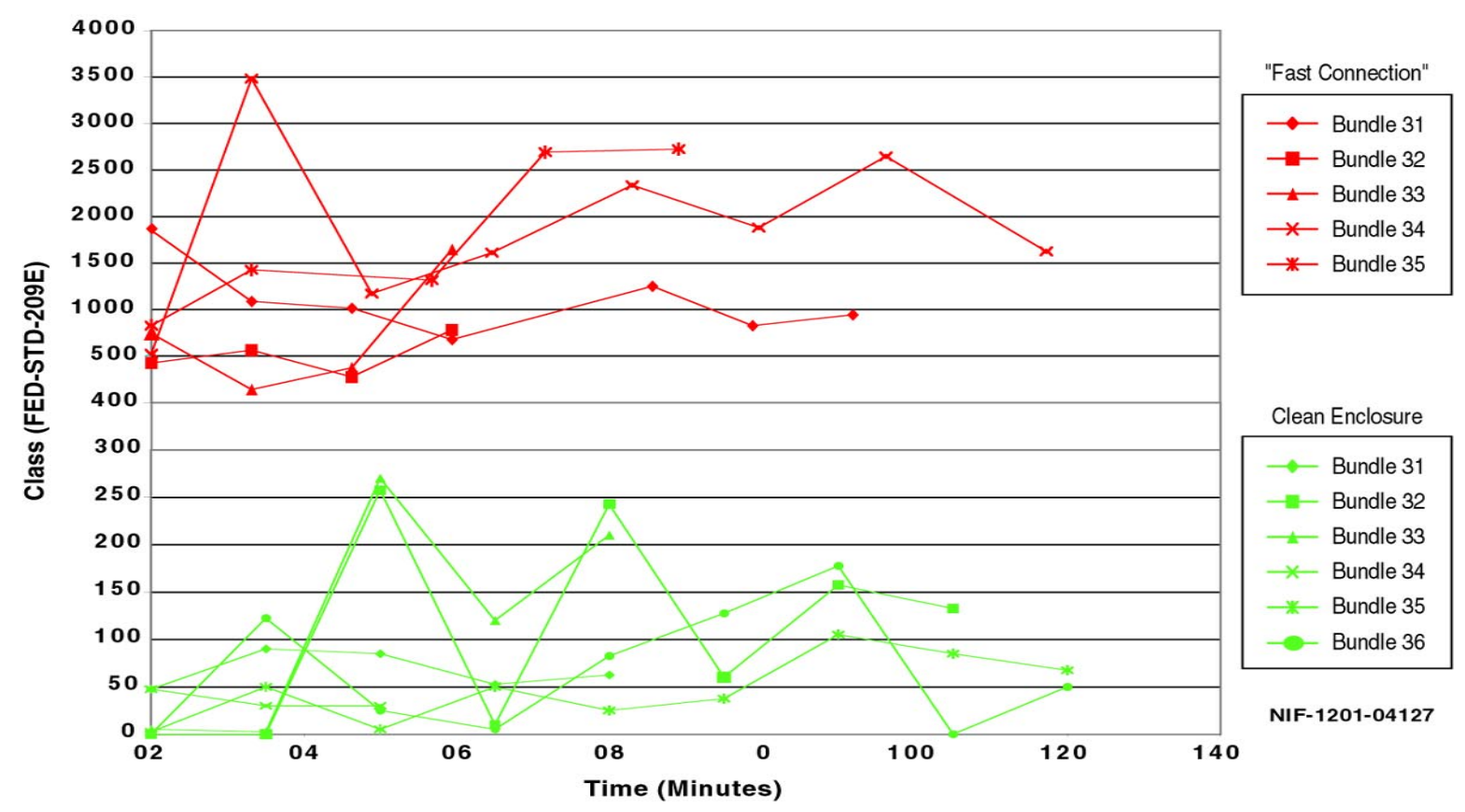

Figure 14. Cleanliness exposure for field-built clean enclosures and fast connections.

During the installation of the Cluster 3 beampath, more than 1,900 surface swipes were collected and exposure monitoring measurements performed for all the subassembly and clean connection efforts. The relationship between the cleanliness requirements defined in Table 1 and the exposure, or classhour, requirements in Table 3 was established based upon the allowable change in surface cleanliness from Level 100 to Level 140. A surface accumulation rate was determined by integrating the product of the aerosol class concentration and the Stoke's velocity for a range of particle sizes. Using a model developed empirically by Hamberg and Tribble, a change from Level 100 to Level 140 could result from exposure to a Class 18,500 aerosol for 1 hour, a Class 1,250 for 8 hours, and a Class 100 for 57 hours. The nonlinearity of the values results from the Hamberg model which states that the surface particle accumulation rate is proportional to the Class ${ }^{0.773} \mathrm{x}$ hours of exposure. The 1,900 swipe measurements taken during installation and shown in Figure 13 clearly indicate that the implementation of the cleanliness protocol procedures has resulted in surface cleanliness levels below Level 140, with the clean assembly being performed in varying construction conditions.

With the completion of the Cluster 3 beampath in one of the laser bays, the beampath components in the switchyard and target bay are being installed, and the clean utility systems are being fabricated, installed, and tested. The cleanliness requirements for the clean utility systems are listed in Table 2 in terms of both particulate and AMC levels, which will be satisfied prior to connecting the utility systems to the beampath. For portions of the beampath with amplifiers, a purge system will be installed that maintains filtered air at a pressure less than $2.5 \mathrm{kPa}$ within the beampath until the CDA system is connected. The purge system will provide air at the connection with the beampath that is consistent with the precision clean piping requirements in Table 2. After integration of the clean beampath and utility systems, the cleanliness of the beampath will be maintained with several systems. Since the portions of the beampath that are subjected to vacuum are most sensitive to AMCs as discussed in Section 1, getter pans with silica-gel will be installed in the end vessels. Since AMCs transport relatively easily in vacuum where they only infrequently collide with gas molecules, these silica-gel getters will maintain the vapor pressure, or maximum concentration, below the sol-gel limit shown in Figure 5 by providing a condensation surface for the AMCs. For portions of the beampath with 
amplifiers, the flashlamps discussed in Section 1 will be fired sequentially and during this amplifier cleaning process, organic films will be removed from the interior surfaces of the beampath due to the high temperatures from the flashlamp fluence of several Joules $/ \mathrm{cm}^{2}$. The aerosol suspension created by these removed films will then be removed by the CDA cooling and purge system.

In order to evaluate the cleanliness of the installed beampath, two systems are being installed to monitor the particulate and AMC levels. For portions of the beampath with amplifiers, the Aerosol Monitoring System (AMS) consists of an airborne particle counter, a cascade impactor for collecting particles, and an AMC detector. The AMS has a low volume gas extraction system that continuously monitors the environment within the beampath in terms of particle size, particle concentration, and molecular constituents. It is expected that the background level in the beampath will be less than Class 10 prior to firing of the amplifier flashlamps and will momentarily increase to Class 10,000 immediately following the firing. The AMC and CDA purge system will operate continuously to maintain the desired cleanliness levels in the beampath. In other portions of the beampath, such as the vacuum vessels, AMC levels will be measured with a Surface Acoustic Wave (SAW) sensor. The SAW detector contains a solgel coated surface that simulates the antireflection characteristics of the lenses. As the sol-gel surface in the SAW absorbs AMCs, the increased mass changes the resonant frequency of the detector providing a measure of the level of AMCs in the environment. Both the AMS and SAW detectors are planned for installation on NIF by mid-2002.

\section{Summary}

To achieve the cleanliness requirements for the beampath interior, a graded CCP approach is used because the less than Class 10 NIF beampath and utilities are being constructed in a stadium-size Class 100,000 building. Installation of the beampath components uses either localized minienvironments of Class 100 or better or ambient conditions less than Class 5,000 for each clean connection. Garment, equipment, and operational considerations are evaluated with process verification. Verification of the beampath and utility cleanliness is achieved by monitoring cleanliness exposure, evaluating particulate levels, and measuring NVR levels. Results from cleanliness verification indicate that the CCP specifications are achieving the NIF cleanliness requirements for the beampath and clean utilities.

\section{Acknowledgments}

This work was performed by University of California Lawrence Livermore National Laboratory under the auspices of the United States Department of Energy, National Nuclear Security Administration, under Contract Number W-7405-ENG-48. The authors thank the following individuals for their technical assistance in preparing this paper: John Honig, James A. Pryatel, Mary L. Spaeth, Charles B. Thorsness, and Donald L. Wadkins.

\section{References}

Busnaina, A.A., Lin, H., "Cleanliness During Assembly of Clean NIF Components," NIF-0063126, Lawrence Livermore National Laboratory, Livermore, California, 2001.

Fair, J., Thorsness, C., "Maximum Acceptable Organic Concentrations in NIF Enclosures Containing Sol-coated Optics," NIF-0039225, Lawrence Livermore National Laboratory, Livermore, California, November 19, 1999. 
Gourdin, W., Thorsness, C., "LTAB Particulate and AMC Requirements Outside of the Beampath," NIF-0067040, Lawrence Livermore National Laboratory, Livermore, California, July 9, 2001.

Gourdin, W.H., Gdowski, G., Ertel, J., "Summary Report: Cavity Spatial Filter Vessel 2, Cluster 2 Entry and Rouge Remediation," UCRL-ID-144967, Lawrence Livermore National Laboratory, Livermore, California, July 19, 2001.

Hamberg, O., "Particle Fallout Predictions for Clean Rooms," Journal of Environmental Science, pp.15-20, May/June 1982.

Institute of Environmental Sciences and Technology, FED-STD-209E, "Airborne Particulate Cleanliness Classes in Cleanrooms and Clean Zones," 940 E. Northwest Highway, Mt. Prospect, Il., September 11, 1992.

Lafave, V., Webster, R., Stowers, I., "Aerospace Prospective on Contamination Control - for the NIF," NIF-0048121, Lawrence Livermore National Laboratory, Livermore, September 18, 2001.

Lawrence Livermore National Laboratory, Specification CSP-16, "Installation of Laser Bay Beam Path Enclosures at Livermore Site, National Ignitition Facility," NIF-5002441-OA, Livermore, California, June, 2000.

Lawrence Livermore National Laboratory, Specification OCS-0104, CSP-19, "Install and Align Beam Enclosures in Switchyard 1 \& 2 at Livermore Site, National Ignition Facility," NIF-5002723-OA, Livermore, California, August 18, 2000.

Lawrence Livermore National Laboratory, Specification OCS-0106, CSP-14B, "Laser Bays, Capacitor Bays \& Switchyards Beampath Infrastructure Utilities at Livermore Site," NIF-5002082-OA, Livermore, California, December 22, 2000.

Marshall, C., Pryatel, J., Sommer, S., Stowers, I., "Cleanliness Requirements for Beampath Utility Systems," NIF-0057299, Lawrence Livermore National Laboratory, Livermore, California, December 5, 2000.

Spaeth, M., Stowers, I., Honig, J., LaTray, D., "Recommended Precision Cleanliness Verification Requirements for Evaluation of Particles in the Beampath Infrastructure of the NIF," NIF-5008147OA, Lawrence Livermore National Laboratory, Livermore, California, May 4, 2001.

Stowers, I.F. and Ravizza, D.L., "The Particle Cleanliness Validation System," UCRL-JC-145932, Lawrence Livermore National Laboratory, Livermore, California, January 2002.

Tribble, A.C., Boyadjian, B., Davis, J., Haffner, J., and McCullough, E., "Contamination Control Engineering Design Guidelines for the Aerospace Community," NASA Contractor Report 4740, National Aeronautics and Space Administration, Marshall Space Flight Center, Alabama, May 1996.

U.S. Department of Defense, MIL-STD-1246C, "Product Cleanliness Levels and Contamination Control Program," April 11, 1994.

Wadkins, D. and LaFave, V., "Evaluation of Hairnet, Face Masks, and Booties for Protocol Level 2," CM99-0086, Lawrence Livermore National Laboratory, Livermore, California, October 29, 1999. 